\title{
A cross cultural review of the ethical issues in dementia care in Kerala, India and The Netherlands
}

\author{
C. V. Sowmini ${ }^{1 *}$ and Raymond De Vries ${ }^{2}$ \\ ${ }^{1}$ Medical Research Officer, HRRC/ICMR, Department of Obstetrics and Gynaecology Medical College, Trivandrum, \\ Kerala, India \\ ${ }^{2}$ Bioethics Program, University of Michigan, School of Medicine, Ann Arbor, MI, USA
}

\section{SUMMARY}

Background The paper explores the diverse ethical issues in the care of persons with dementia, in the Netherlands and Kerala, India. These cross-cultural data are used to suggest newer ways for addressing the ethical issues in a mutually enhancing manner.

Methods A thorough review of the literature focusing on ethical aspects of the care for persons with dementia.

Results The medical paradigm is dominant in the Netherlands and awareness of dementia as an organic brain disease is low in Kerala. Institutionalized care is more common in the Netherlands and home-based care is the norm in Kerala. Institutional care is costly, whereas home-based care is stressful for caregivers.

The advanced directive plays an influential role in the Netherlands, but this mechanism is yet to evolve in Kerala.

The legal and social setting of the Netherlands has a strong influence on physician decision-making concerning end of life issues. In Kerala, discussion of these matters is nearly unknown.

Conclusion Limited awareness of dementia in Kerala should be addressed in public forums, which can then be used to garner governmental support. The predominantly institutional model of care-giving in the Netherlands and home-based caregiving in Kerala, each have their strengths; policy makers in both societies can usefully apply the values and merits inherent in both models.

A culturally appropriate implementation of the advanced directive will have beneficial medical, social, and economic impacts in Kerala.

The remarkable disparity between the Netherlands and Kerala in dealing with end-of-life issues will allow more philosophically and socially informed ways of addressing the ethical questions that arise in those situations. Copyright (C) 2008 John Wiley \& Sons, Ltd.

KEY WORDS — dementia Kerala; dementia Netherlands; advanced directive; care givers strain; end of life decision-making; palliative care; ethics

\section{INTRODUCTION}

Culture plays a significant role in shaping the perceptions and experiences of disease; nowhere is this more true than in dementia, a disease involving

*Correspondence to: Dr C. V. Sowmini, GRA 126, Gowreesapattom, Pattom Palace PO, Trivandrum, 695004, Kerala, India.

E-mail: sowminicv@gmail.com

Copyright (C) 2008 John Wiley \& Sons, Ltd. both the body and the mind. Analysis of the ethical issues related to dementia is coloured and controlled by cultural factors, especially as ethics itself is a culturally positioned and conditioned phenomenon. Though the cultural embeddedness of ethics is a major methodological challenge to comparative research (10/66 Dementia Research Group, 2000), the two cultures studied here-Kerala, a state in the southern India, and the Netherlands - have sufficient common- 
alities, in terms of aging population, dementia prevalence and care giving settings, to make comparison possible and productive.

\section{The proximate setting of the comparative references}

Both Kerela and the Netherlands have rapidly increasing elderly populations. Life expectancy at birth in Kerela is 73 years (Human Development Index), compared to 79 years in the Netherlands (World Fact Book, 2007). Ten percent of the population in Kerela is aged 60 years and above, an indication of considerable increase in longevity there (Shaji et al., 2002; Shaji et al., 2005). Increased life expectancy brings with it a higher lifetime risk (Reisberg et al., 1982) of dementia in both regions, presenting challenges to the people and institutions that care for the elderly. In Kerela, rapid demographic transition coupled with modernization has upset traditional forms of care, leading to a move away from care given by the extended family to a system much like the Netherlands where the nuclear family is responsible for care.

\section{METHODS}

The method used for our comparison is a review of the literature dealing with ethical issues related to dementia. There are a few published articles on dementia care in Kerela, and only a few of those deal with ethical issues. For this reason the 11 articles in our survey are not restricted to those focusing only on ethical issues. Not surprisingly, there are a huge number of articles examining the ethical issues of dementia care in the Netherlands; we chose the 26 most-cited for this analysis.

\section{RESULTS}

\section{Empirical findings}

The burden of disease. In Kerala, the prevalence of dementia in urban population is 33.6/1000 (Shaji et al., 2005); in the Netherlands the prevalence is 11.3/ 1000 (Hofman et al., 1991; Ferri et al., 2005). Family history is a reported risk factor for Alzheimer's disease and hypertension is regarded as risk factor for vascular dementia (Shaji et al., 2005). Behavioral symptoms were reported in $71 \%$ of those with mild to moderate dementia in Kerala (10/66 Dementia Research Group, 2004: 455). Behavioral and psychological symptoms of dementia (BPSD) are independent predictors for caregivers' burden. In the Netherlands, more than $80 \%$ of dementia patients in nursing homes reported at least one clinically significant neuropsychiatric symptom (Zuidema et al., 2007). Agitated behaviours have a common basis in different settings (Zuidema et al., 2007).

The setting. In the Netherlands, nursing home practice is a specialty for which physicians are trained exclusively in a nursing home setting (Hoek et al., 2001; Helton et al., 2006). Eighty-two percent of those diagnosed with dementia had used one or more types of formal care and $55 \%$ were institutionalized (Boersma et al., 1998). Age, severity of dementia, somatic disorders, dependency in the form of activities of daily living (ADL) and marital status were predictors for high levels of care. Whereas in Kerala home based care is predominant.

The cost of care-giving. In the Netherlands, the cost of dementia care is a severe burden at national level (Goes et al., 1998). Yearly 1.6 billion US\$ are spent on dementia care. The cost per day for treatment in a Dutch nursing home is 146 US\$. Cost estimation at institutional level is not yet an issue in Kerala because of predominant home based care.

The care givers' perspective. In Kerala, the majority of care givers are daughters-in-law of women with dementia. Behavioural problems and incontinence were the chief sources of caregivers' strain. The majority of caregivers experienced significant deterioration in their mental health (Shaji et al., 2003). Whereas, in the Netherlands, professionals support both the patient and the care giver (Kümpers et al., 2005). These professionals explicitly consider the feelings of families while giving proper respect to the autonomy of the patient (Helton et al., 2006; Koopmans et al., 2007); nursing homes are organized to allow maximum freedom to residents with dementia (Kalis et al., 2005: 40; Riedijk et al., 2006).

Awareness of dementia. In Kerala, dementia is considered as a natural consequence of aging and hence there is only limited of it being aware as an organic brain syndrome. Caregivers at time tend to misinterpret the behaviour of the dementia patients as deliberate (Shaji et al., 2003). The resultant refusal to seek adequate medical care poses a challenge to public health in Kerala (Shaji et al., 2002; Shaji et al., 2003). However, public health officials are promoting early diagnosis, using locally developed and culturally 
adapted tools for case detection (Mathuranath et al., 2004; Iype et al., 2006). In the Netherlands recent research is helping general practitioners to detect cognitive impairment, enabling patients and care givers to organize future care and to benefit from facilities that offer information and support when patient's insight is still relatively preserved (Jansen et al., 2007).

\section{Ethical findings}

Advance Directive (AD) and durable power of attorney. Differences in the use of advance directives reveal significant differences in the societies studied here. An advance directive (AD) is an extension of a competent patient's moral and legal right to refuse treatment in the post-competent period (Berghmans et al., 1997). In the Dutch setting the advance directive-based on the principle of respect for autonomy and individual self-determination-promotes explicit communication about the hastening of death without fear of litigation. Good rapport and communication between the physician, the patient, and the patient's family in the formal care setting in the Netherlands make the use of an AD a formality. However, advance directives are not without serious ethical problems (Berghmans, 1997; Van Delden, 2007). These include: lack of correspondence between the $\mathrm{AD}$ and the eventual decisions of the patients, incompatibility between the formulation of the $\mathrm{AD}$ and the actual point of its implementation (in terms of time, scientific knowledge, and therapeutic options), and lack of clarity about why and when an AD can be reconsidered. ADs fail to take into account the remarkable ability of humans to adapt to changing life situations and the coping mechanisms that allow humans to redefine circumstances once seen as distressing. ADs cannot adequately account for the discontinuity of the person: the 'self' who wrote the directive before the advent of dementia may or may not be the same 'self' who presently suffers from dementia.

In Kerala, patients with dementia very rarely use an advance directive. This idea of the advance directive, common in Western, industrialized societies, has not yet trickled down to ethical discussions on dementia in Kerala. We found only one study that explored the benefit of having a durable power of attorney to help settle the will, bank accounts, and the distribution of pension benefits (Shaji and Iype, 2003).

End of life decisions in dementia cases. The Royal Dutch Medical Society identifies four kinds of life shortening actions near end of life, viz., withholding treatment, stopping treatment, treatment of symptoms accompanied by shortening of life (the principle of double effect) and actions that terminate life (i.e. administering lethal drugs) (Cusveller and Henk, 1996). Various ethicists offer elaborate arguments about the conditions under which such measures could be used (Berghmans, 1998; Van Delden, 2007). According to the Royal Dutch Medical Society, the use of medical treatments at the end of life is to be determined and justified by considering: (1) the supposed (or reconstructed) will of the patient; (2) the burden of the treatment to be given or considered; (3) the positive effect of treatment and (4) the interests of the patient (Cusveller and Henk, 1996).

Foregoing artificial nutrition and hydration. In the Netherlands, the decision to forego Artificial Nutrition and Hydration (ANH) is made relatively frequently by nursing home physicians. ANH were totally avoided in $40 \%$ of deaths of incompetent patients in the Dutch nursing homes. In $90 \%$ of cases an agreement was made about ANH duration before the start of ANH. Nurses were consulted in $85 \%$ of cases regarding the use of ANH, and the family members in $74 \%$ of cases. The decision was unanimous in $88 \%$ of cases. In the Netherlands the incidence of starting ANH in nursing home patients with dementia is as low as 3/100 patients a year (Onwuteaka-Philipsen et al., 2001; Pasman et al., 2004; Van Wigcheren et al., 2007). A survey of the cause of death in Dutch nursing homes showed $35.2 \%$ of deaths attributed to cachexia/ dehydration, $20.9 \%$ to cardiovascular disorders, and $20.1 \%$ to pneumonia (Koopmans et al., 2007).

Regarding end of life decision-making and care plans for dementia patients in Kerala, none of the literature discusses euthanasia, the withholding or withdrawing of treatment, or decisions about the use of ANH at the terminal stages of illness. There are no recorded reports of the practice of Euthanasia and it is not legalized in Kerala or India. In general all possible treatments are provided to sustain life.

Several articles from the Netherlands and only one from Kerala discuss the option of palliative care in dementia (Hughes et al., 2005; Lloyd-Williams et al., 2005; Shaji, 2005; Treloar et al., 2005).

\section{DISCUSSION}

The above analysis and identification of the ethical issues related to dementia in a cross cultural setting 
suggests ways that each society may borrow from the other in an effort to promote better care.

\section{Perception of dementia - from the natural aging to the organic brain disease}

This comparative study warrants a perceptual redefining of the understanding of dementia in both regions. In Kerala, there is little awareness of dementia as an organic brain disease or acknowledgement of the pathological and medical dimensions of the illness; rather dementia is considered to be a natural aging process (Shaji et al., 2003). Hence people tend to be reluctant to seek health care and the health care system is not adequately equipped to address their needs. For the state as a whole, dementia is emerging as a public health challenge with considerable unmet need and many possibilities for intervention. Meanwhile, in the Netherlands, there is a tendency to overvalue the medical dimension of dementia resulting in an approach to the disease dominated by medical treatment. In this respect Kerela has something to teach the Netherlands. The Dutch system could benefit from a more holistic model that emphasizes the relational dimension of care and the value of homebased care.

\section{Care setting - from the institutional to the relational}

The formal and institutional care setting in the Netherlands (Boersma et al., 1998) offers the possibility of a different paradigm for care giving in for Kerala, a paradigm that addresses the neglected aspect of the burden placed on caregivers in homebased care. As K. S. Shaji has rightly observed, there is a wrong assumption that dementia care giving is easier in developing countries because of the larger family size. (Shaji et al., 2003). Although the institutionalized style of care giving does not quite 'fit' with the ethos and worldviews of the traditional culture of Kerala, the emergence of the 'day care' centres in Kerala provide some balanced alternatives (Shaji and Iype, 2003). Respite care and community-based dementia care, if developed, will reduce the caregivers strain.

A balancing of the institutional and the familial, the formal and the relational, and incorporating respect for the deeper personal and emotional dimensions of the patient would not only enhance the care model in Netherlands, it would also reduce the alarmingly high cost of caring in the Netherlands.
Advance directive - from the deterministic to the qualified

Careful adaptation of the ethically useful tool of the advance directive as practiced in the Netherlands could help solve several ethical and social confusions related to care of the person with dementia in Kerala. This is especially true given the absence of an established health insurance system in Kerala, which places the cost of treatment on patients and families and results in limited care seeking in Kerala (Wolstenholme et al., 2002; Shaji et al., 2003). An advance directive will also facilitate proper medical management for those with advanced disease and act as a guard against abuse of the elderly.

On the other hand the overly-determined nature of the advance directive in the Netherlands could be moderated by paying attention to the way the relational embeddedness of the person in the culture of Kerala promotes an atmosphere of care that is gratifying and comprehensive. This perspective would encourage a case-by-case, specific approach in the execution of advance directives.

\section{End of life-from the paternalistic to the dialogical}

The traditionally paternalistic model of medical practice in Kerala, could benefit by the adoption of certain features of Dutch medicine. More emphasis on the autonomy of the patient will have tangible implications for a different approach in the caring of the demented, ensuring the significance of a 'dialogical' approach, rather than a one-way conversation, in decision-making. Enhanced autonomy (Quill and Brody, 1996) will lead to increased awareness, better interaction and communication (Zarit et al., 1999) with family members, and greater involvement on the part of the wider community of health care professionals.

The Dutch model also encourages non-aggressive treatment as a more appropriate option in medically futile conditions, perhaps tempering the aggressive commitment of physicians in Kerala to the prolongation of life. For their part, Dutch physicians might use the more aggressive resistance to death found in Kerala to rethink their approach to artificial nutrition and hydration (Van Wigcheren et al., 2007).

\section{Euthanasia-from the physical to the existential}

The striking similarities in the demographic trends of the two regions under study belie a remarkable difference in the approach to the end of life in both 


\section{KEY POINTS}

- Adequate awareness of dementia as a culturallysituated, multi-faceted illness must be given greater importance in dealing with the ethical issues associated with the disease.

- Cross-cultural study suggests a revised model of care giving that ensures the quality of life for both patients and caregivers.

- The use of advance directives is shaped by both cultural ideas and social resources.

- Treating ethical issues in dementia in a comprehensive manner requires attention to philosophical theory, cultural values, and social resources.

regions. Despite the stronger gravity of dementia in terms of the quality of life and the quantity of the patients in Kerala, the issue of euthanasia remains totally alien there. This is the result of the particular worldview and belief-system of the people of Kerala with its concern for the relational and spiritual dimension of human life. In the far more secular Netherlands, euthanasia is seen as a physical matter, a strategy to reduce suffering. Looking to Kerela, and the relationship between religious ideas and medical practices highlights the need to consider how medical decisions about the care of the elderly are significantly shaped by non-medical (i.e. cultural and religious) factors.

\section{CONFLICT OF INTEREST}

None known.

\section{AUTHORS' ROLES}

C. V. Sowmini was responsible for formulating research question, design of the study, carrying out data collection and analyzing the data and writing the paper. Raymond De Vries was responsible for formulating research question, design of the study and critically revising the scientific content of the paper.

\section{ACKNOWLEDGEMENTS}

This paper is the result of research performed as part of the educational programme Erasmus Mundus Master of Bioethics.

\section{REFERENCES}

Berghmans RLP. 1998. Ethics of End-of- Life decision in cases of dementia: view of the Royal Dutch Medical Association with some critical comments. Alzheim Dis Assoc Disord 13: 91-95.

Berghmans RLP. 1997. Advance Directive and dementia. Ann NY Acad Sci. 105-110.

Boersma F, Eefsting JA, Van Den Brink W, Van Tilburg W. 1998. Care services for dementia patients: predictors for service utilization. Int J Geriatr Psychiatry 12: 1119-1126.

Cusveller B, Henk J. 1996. Life terminating actions with severely demented patients: a critical assessment of a report of the Royal Dutch Society of Medicine. Issues Law Med 12: 1996.

Ferri CL, Prince M, Brayne C, et al. 2005. Global prevalence of dementia: a Delphi consensus study. Lancet 366: 2112 2117.

Goes E, Blom M, van der Roer N. 1998. Costs of dementia in the Netherlands. Int Soc Technol Assess Health Care Int Soc Technol Assess Health Care Meet 14: 74.

Helton MR, van der Steen JT, Daaleman TP, et al. 2006. A crosscultural study of physician treatment decisions for demented nursing home patient who develop pneumonia. Ann Fam Med 4: 221-227.

Hoek JF, Ribbe MW, Hertogh CMPM, van der Leuten CPM. 2001. The specialist training program for nursing home physicians: a new professional challenge. J Am Med Dir Assoc 2: 326330.

Hofman A, Rocca WA, Brayne C, et al. 1991. The prevalence of dementia in Europe: a collaborative study of 1980-1990 findings. Int J Epidemiol 20(3): 736-748.

Hughes JC, Robinson L, Volicer L. 2005. Specialist palliative care in dementia Specialised units with outreach and liaison are needed. BMJ 330: 57-58.

Iype T, Ajitha BK, Antony P, et al. 2006. Usefulness of Rowland Universal Dementia Assessment Scale in south India. J Neurol Neurosurg Psychiatry 77: 513-514.

Jansen APD, van Hout HPJ, Nijpels G, van Marwijk HWJ, de Vet HCW, Stalman WAB. 2007. Yield of a new method to detect cognitive impairment in general practise. Int J Geriatr Psychiatry 22(6): 590-597.

Kalis A, Schermer MHN, van Delden JJM. 2005. Ideals regarding a good life for nursing home residents with dementia: view of professional caregivers. Nursing Ethics 12: 30-42.

Koopmans RTCM, van der Sterren KJMA, van der Steen JT. 2007. The 'natural' end point of dementia: death from cachexia or dehydration following palliative care? Int J Geriatr Psychiatry 22: $350-355$.

Kümpers S, Mur I, Maarse H, van Raak . 2005. A comparative study of dementia care in England and the Netherlands using neoinstitutionalist perspectives. Qualitat Health Res. 15: 11991230 .

Lloyd-Williams M, Payne S, Dennis M. 2005. Specialist palliative care in dementia: Patients with dementia are unable to access appropriate palliative care. BMJ 330: 671-672.

Mathuranath PS, Hodge JR, Mathew R, et al. 2004. Adaptation of the ACE for a Malayalam speaking population in southern India. Int J Geriatr Psychiatry 19: 1188-1194.

Onwuteaka-Philipsen BD, Pasman HR, Kruit A, et al. 2001. Withholding or withdrawing artificial administration of food and fluids in nursing home patients. Age Aging 30: 459-465.

Pasman HR, Onwuteaka-Philipsen BD, Ooms ME, et al. 2004. Forgoing artificial nutrition and hydration in nursing home patients with dementia: patients, decision making and participants. Alzheimers Dis Assoc Disord 18: 154-162. 
Quill TE, Brody H. 1996. Physicains recommendations and patients autonomy: finding a balance between physician power and patient choice. Ann Intern Med 125: 763769.

Reisberg B, Ferris SH, de Leon MJ, Crook T. 1982. The Global Deterioration Scale for assessment of primary degenerative dementia. Am J Psychiatry 139: 1136-1139.

Riedijk SR, De Vugt ME, Duivenvoorden HJ, et al. 2006. A caregiver burden, health-related quality of life and coping in dementia caregivers: a comparison of frontotemporal dementia and Alzheimer's disease. Dement Geriatr Cogn Disord 22: 405412.

Shaji KS, Arun Kishore NR, Praveen Lal K, Prince M. 2002 Revealing a hidden problem. An evaluation of a community dementia case - finding programs from the Indian 10/66 dementia research network. Int $J$ Geriatr Psychiatry 17: 222225.

Shaji KS, Smitha K, Praveen Lal K, Prince MJ. 2003. Caregivers of people with Alzheimers disease: a qualitative study from the Indian 10/66 Dementia Research Network. Int J Geriatr Psychiatry 18: 1-6.

Shaji KS, Iype T. 2003. Management of dementia: a developing country scenario. In Reviews in Indian Neurology, Radhakrishnan K (ed.). SCTIMST: Trivandrum, Kerala.

Shaji S, Bose S, Verghese A. 2005. Prevalence of dementia in urban populations in Kerala, India. Br J Psychiatry 186: 136-140.

Shaji KS. 2005. Specialist palliative care in dementia: home based palliative care is important in developing countries. BMJ 330: 672.
10/66 Dementia Research Group. 2000. Methodological issues for population based research into dementia in developing countries. Int J Geriatr Psychiatry 15: 21-30.

10/66 Dementia Research Group. 2004. Behavior and psychological symptoms of dementia in developing countries. Int Psychogeriatr 16(4): 441-459.

Treloar A, Newport J, Venn-Treloar J. 2005. Specialist palliative care in dementia: and hope for terminal care at home is needed in developed countries. BMJ 330: 672

Van Delden JJM. 2007. The unfeasibility of requests for euthanasia in Advance Directives. $J$ Med Ethics 30: 447-452.

Van Wigcheren PT, Onwuteaka-Philipsen BD, Pasman HRW, et al. 2007. Starting artificial nutrition and hydration in patient with dementia in the Netherlands: ferquencies, patient characteristics and decision making process. Aging Clin Exp Res 19(1): 26-33.

Wolstenholme JI, Fenn P, Gray AM, et al. 2002. Estimating the relationship between disease progression and cost of care in dementia. Br J Psychiatry 181: 36-42.

Zarit SH, Gaugler JE, Jarrott SE. 1999. Useful services for families: research findings and directions. Int J Geriatr Psychiatry 14(3): $165-178$.

Zuidema SU, Derksen E, Verhey FRJ, Koopmans RTCM. 2007. Prevalence of neuropsychiatric symptoms in a large sample of Dutch nursing home patients with dementia. Int J Geriatr Psychiatry 20: 41-49.

Zuidema SU, de Jonghe JFM, Verhey FRJ, Koopmans RTCM. 2007. Agitation in Dutch institutionalized patients with dementia: factor analysis of Dutch version of the Cohen-Mansfield Agitation Inventory. Dement Geriatr Cogn Disord 23: 35-41. 